\title{
Editorial: Outstanding PRST-AB Articles from 2011
}

Following the 2010 initiative of PRST-AB Editorial Board Member Vladimir Shiltsev, seven outstanding PRST-AB papers from the year 2011 have been selected and approved by the PRST-AB Editors with input provided from the Editorial Board. The selection criteria included the original referee assessments, the number of citations since publication, and the editors' own impression of a paper's importance. The selected PRST-AB articles from 2011 provide a well balanced picture of PRST-AB coverage:

1. S. Roling, B. Siemer, M. Wöstmann, H. Zacharias, R. Mitzner, A. Singer, K. Tiedtke, and I. A. Vartanyants, Temporal and spatial coherence properties of free-electron-laser pulses in the extreme ultraviolet regime, Phys. Rev. ST Accel. Beams 14, 080701 (2011).

2. Chao Feng, Tong Zhang, Jianhui Chen, Haixiao Deng, Meng Zhang, Xingtao Wang, Bo Liu, Taihe Lan, Dong Wang, and Zhentang Zhao, Measurement of the average local energy spread of electron beam via coherent harmonic generation, Phys. Rev. ST Accel. Beams 14, 090701 (2011).

3. Wolfgang Weingarten, Field-dependent surface resistance for superconducting niobium accelerating cavities, Phys. Rev. ST Accel. Beams 14, 101002 (2011).

4. P. Piot, Y.-E Sun, J. G. Power, and M. Rihaoui, Generation of relativistic electron bunches with arbitrary current distribution via transverse-to-longitudinal phase space exchange, Phys. Rev. ST Accel. Beams 14, 022801 (2011).

5. A. C. Dexter, G. Burt, R. G. Carter, I. Tahir, H. Wang, K. Davis, and R. Rimmer, First demonstration and performance of an injection locked continuous wave magnetron to phase control a superconducting cavity, Phys. Rev. ST Accel. Beams 14, 032001 (2011).

6. N. V. Elkina, A. M. Fedotov, I. Yu. Kostyukov, M. V. Legkov, N. B. Narozhny, E. N. Nerush, and H. Ruhl, QED cascades induced by circularly polarized laser fields, Phys. Rev. ST Accel. Beams 14, 054401 (2011).

7. Dmitry Shatilov, Eugene Levichev, Evgeny Simonov, and Mikhail Zobov, Application of frequency map analysis to beam-beam effects study in crab waist collision scheme, Phys. Rev. ST Accel. Beams 14, 014001 (2011).

This identification of seven key articles from 2011 represents another step towards bringing important papers to the attention of our readers. Improved mechanisms and modes for highlighting outstanding PRST-AB articles are presently under development.

Frank Zimmermann

Editor

Published 21 December 2012

PACS numbers: 01.30.Ww

Published by the American Physical Society under the terms of the Creative Commons Attribution 3.0 License. Further distribution of this work must maintain attribution to the author(s) and the published article's title, journal citation, and DOI. 